\title{
Self-consistency and coherent effects in nonlinear resonances
}

\author{
I. Hofmann*, G. Franchetti*, J. Qiang ${ }^{\dagger}$ and R.D. Ryne ${ }^{\dagger}$ \\ ${ }^{*}$ GSI, 64291 Darmstadt, Germany \\ ${ }^{\dagger}$ LBNL, 94720 Berkeley, USA
}

\begin{abstract}
.
The influence of space charge on emittance growth is studied in simulations of a coasting beam exposed to a strong octupolar perturbation in an otherwise linear lattice, and under stationary parameters. We explore the importance of selfconsistency by comparing results with a non-self-consistent model, where the space charge electric field is kept "frozen-in" to its initial values. For Gaussian distribution functions we find that the "frozen-in" model results in a good approximation of the self-consistent model, hence coherent response is practically absent and the emittance growth is self-limiting due to space charge de-tuning. For KV or waterbag distributions, instead, strong coherent response is found, which we explain in terms of absence of Landau damping.
\end{abstract}

\section{INTRODUCTION}

The effect of space charge on nonlinear resonances has received increasing attention with the need of optimizing the performance of storage rings or synchrotrons with high intensity or high phase space density. In a recent experiment carried out at the CERN proton synchrotron (see companion paper to this conference, Ref. [1]) the 3D aspects of the resonance effect of a single octupole on an $1 \mathrm{~s}$ long injection flat-bottom have been explored by simulation as part of a code bench-marking effort by abandoning self-consistency and using an analytical solution of Poisson's equation for the "frozen-in" density profile. The present study is carried out in order to advance our understanding of the importance of self-consistency as well as the differences in resonance behavior of coasting and bunched beams. We adopt similar parameters as in Ref. [1], like a fixed working point varying over an interval above the fourth order resonance at $Q_{x}=6.25$, as well as comparable space charge tune shifts.

The most obvious effect of space charge is the incoherent tune shift and spread, which leads to an extended foot-print of single particle tunes in the tune diagram. Less obvious is the observation that the response on a resonance may be modified by the coherent motion of all, or of a large fraction of particles, which is the main expression of self-consistency. It leads to an additional time-dependent force, which must be added to the external forces, and which may cause a coherent shift of the resonance condition. This phenomenon was studied in some detail for second order resonances in connection with the Spallation Neutron Source Ring, where it was found that this coherent shift has a favorable effect on the tolerable intensity [2]. The present study, however, shows that for Gaussian beams and fourth order resonances such a coherent effect is practically absent.

\section{SIMULATION}

We use the self-consistent 2D particle-in-cell (PIC) version of the MICROMAP code [3] with $10^{5}$ simulation particles and employing different distribution functions on a $64 \times 64$ grid filling a rectangular boundary of $70 \times$ $70 \mathrm{~mm}$ size. In most examples intensity is normalized to a maximum space charge tune shift $\Delta Q_{x}=0.09$ for the Gaussian distribution, and rms equivalence for all others, which implies $\Delta Q_{x}=0.045$ for the equivalent KVbeam. Before discussing the effect of space charge we consider the octupole effect alone. While the experiment was done with 40 A octupole excitation, we find it convenient in this $2 \mathrm{D}$ study to raise the octupole strength by a factor 2.5 (corresponding to $100 \mathrm{~A}$ ) in order to enhance the otherwise weak effects. The normalized strength (in $\mathrm{m}^{-3}$ ) is given here as

$$
K_{3}=1.215 \cdot I
$$

where $I[A]$ is the octupole excitation current.

For a Gaussian distribution we find that the response in terms of rms emittance growth is $24 \%$ for not too strong octupoles, independent of the octupole strength . This is due related to the fact that the stabilizing de-tuning by the octupole increases simultaneously with the resonance driving term. The time required to reach the maximum emittance growth is, however, about inversely proportional to the octupole strength $\left(\approx 10^{3}\right.$ betatron periods 
for $100 \mathrm{~A})$. The response curve as a function of machine tune with clear de-tuning shift is shown in Fig. 1 for a Gaussian distribution. Due to the strong octupole there is

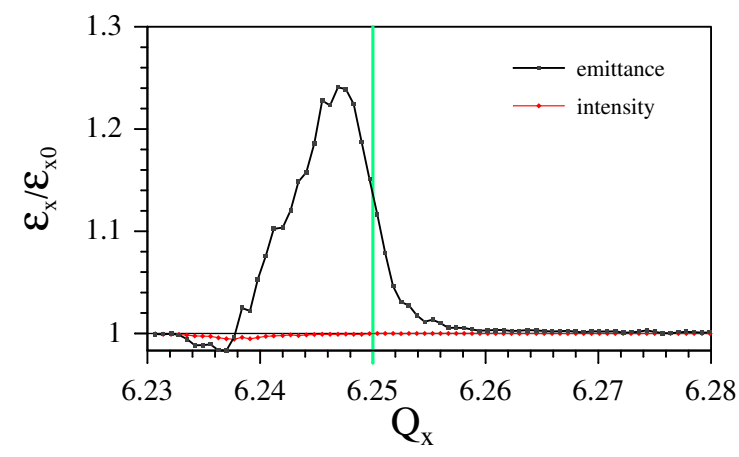

FIGURE 1. 2D simulation without space charge showing relative rms emittance growth and intensity from octupole (100 A).

a visible $(<1 \%)$ beam loss effect for $6.235<Q_{x}<6.24$. This is accompanied by some emittance reduction due to extraction of the larger amplitude particles, which is absent for a 40 A octupole. In order to interpret loss effects we have first carried out a simplified numerical study on the dynamic aperture by searching the maximum stable radius of test particles placed into 20 different directions in the upper half of the $x-y$ plane. Assuming a $200 \mathrm{~A}$ octupole current we have calculated, in the absence of space charge, that the dynamic aperture shrinks to a radius of $2.5 \sigma$ near $Q_{x}=6.25$ for $10^{3}$ turns, and to about $2.2 \sigma$ for $10^{5}$ turns. Comparing several octupole strengths we have found the approximate scaling

$$
D A \approx 35 / \sqrt{K_{3}}
$$

for the dynamic aperture $D A$ expressed in units of $\sigma$.

\section{Distribution function and Landau damping}

Including space charge we obtain results, which depend sensitively on the distribution function. The KVbeam response after 1000 turns is shown in Fig. 2. In order to appreciate the coherent nature of this response we first discuss the rms emittance growth for a "frozenin" space charge electric field, where the initial values are not updated, hence the response is entirely incoherent. The response is non-zero for a distance of the bare machine tune from the resonance line less than 0.045, which equals the incoherent space charge tune shift common to all particles - of the initial KV-beam. Similar to Fig. 1 we observe in the "frozen" response a stopband width of $\approx 0.01$ and a shift from the ideal resonance condition, which is now at $Q_{x}=6.295$. Both effects are a result of the resonance broadening due to the

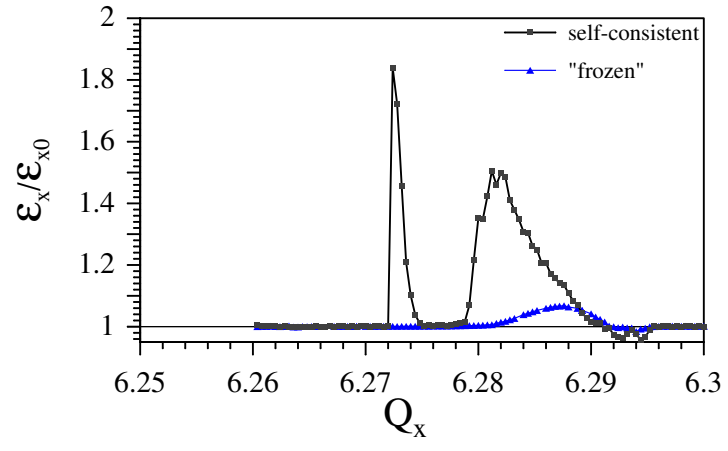

FIGURE 2. 2D simulation of $\mathrm{KV}$ distribution with $\Delta Q_{x}=$ 0.045 and octupole $(100 \mathrm{~A})$ as function of bare machine tune, showing self-consistent and "frozen-in" models (after 1000 turns).

strong octupole force. There is, however, a significant additional de-tuning by space charge, which limits the maximum emittance growth to $<6 \%$ compared with the $25 \%$ growth without space charge.

The self-consistent simulation, instead, shows two high separate peaks split and shifted by coherent space charge effects. The broader peak is interpreted as direct result of the fourth order resonance, but with a coherent tune shift, which suggest that the resonance can be approached roughly $20 \%$ more than suggested by the single-particle response of the "frozen-in" model. The height of this peak exceeds significantly (more than five times) the maximum "frozen-in" response - a pronounced coherent effect. The striking and unexpected large peak at $Q_{x}=6.273$ cannot be explained as direct result of the octupole, but is associated with an envelope instability. Such an envelope instability requires a fractional phase advance of the envelope of half an integer relative to the lattice periodicity as was shown in Refs. [2, 4]. This condition is analogous to the envelope instability in linear accelerators, where a single-particle phase advance above $90^{\circ}$ per focusing period may induce a half-integer unstable envelope. In the present case the "structure period" cannot stem from the smooth first order lattice, but only from the local perturbation induced by the relatively strong octupole. The latter occurs at only one position on the circumference, hence the total phase advance of particles per turn is exceeding $6 \times 360^{\circ}+90^{\circ}$.

To distinguish between second and fourth order resonance effects the coherent coupled mode coefficients $C_{m k}$ introduced in Ref. [5], and elaborated in more detail in Ref. [6], are useful. In a pure single-particle picture it is assumed that the bare machine tune should stay above the resonance by at least the incoherent tune shift. From the point of view of coherent resonance crossing this critical distance is multiplied by $C_{m k}$, which is smaller than unity in most cases. The $C_{m k}$ thus reflect the fact that 
the additional coherent space charge force partly compensates the stationary space charge force and - depending on the order of the resonant mode - allows to bring the bare machine tune closer to the resonance than could be inferred from the single-particle point of view. From the position of the left peak of Fig. 2 we calculate that $C=0.023 / 0.045 \approx 0.5$, which is typical for the envelope breathing mode [6].

For the rms equivalent waterbag distribution in Fig. 3 we find that the envelope instability peak is unchanged. The fourth order resonance effect is visibly reduced, however. We explain this as a weakening of the coherence induced by the finite tune spread. Note that the "frozen-in" emittance response reflects the distribution of single-particle tunes, which is the origin of Landau damping. From Fig. 3 we note that the "frozen-in" curve has no overlap with the coherent tune of the envelope instability (marked by the location of the strong peak) similar to Fig. 2, which therefore cannot be "Landaudamped"; the overlap with the fourth order resonance visibly weakens the coherent response.

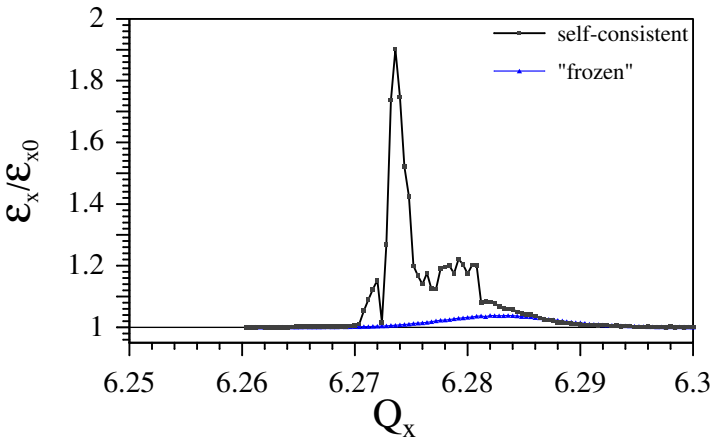

FIGURE 3. 2D simulation of waterbag distribution (same parameters as Fig. 2).

For the Gaussian distribution the picture is essentially different. The much broadened single-particle spectrum of the Gaussian fully overlaps the position of the expected envelope instability frequency, which is therefore effectively "Landau-damped". The much broadened direct response curve is only slightly enhanced compared with the "frozen-in" model (Fig. 4), hence there is an almost complete suppression of the coherent resonance effect. There is also a region of about $0.2 \%$ loss, which is practically identical for the self-consistent and "frozenin" model, and is caused by the extended Gaussian tails.

\section{Dependence on octupole strength}

The strong reduction of emittance growth, caused by the suppression of coherent resonance response for the Gaussian beam, is studied in Fig. 5 as function of octupole strength. The graph shows the relative rms emit-

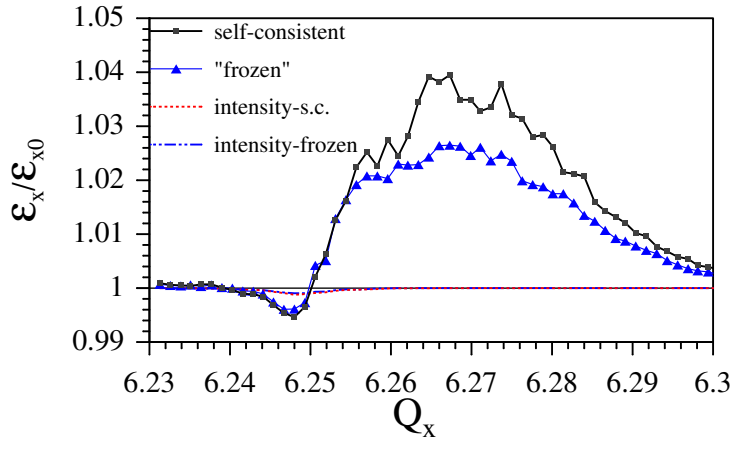

FIGURE 4. 2D simulation of Gaussian distribution (same parameters as Fig. 2, note changed scales).

tance growth and intensity for $Q_{x}=6.27$ obtained from a simulation, where the frozen-in space charge was calculated by an analytical formula valid for round beams (for faster parameter scans, and justified by section ), i.e. equal emittances. Note the drastic suppression of the rms emittance growth compared with the $24 \%$ of the zero-space-charge level. The suppression is most visible for small octupole strength, where the space charge detuning is strongly dominant over the resonance excitation. For octupole strengths above 100 A the shrinking of the dynamic aperture - consistent with Eq. 2 - causes loss from the Gaussian tails, which makes the effective rms emittance shrink even below the starting value.

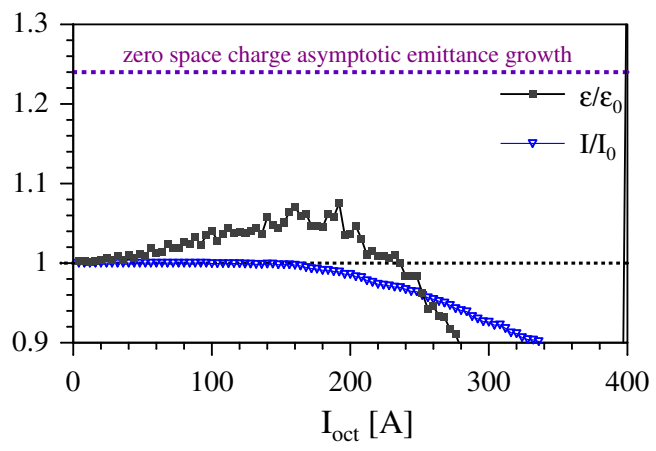

FIGURE 5. 2D simulation of dependence of rms emittance growth and intensity on octupole strength for Gaussian distribution (same space charge shift as Fig. 2).

\section{CODE COMPARISON AND LONG-TERM EFFECTS}

We have compared the output from the MICROMAP code using $10^{5}$ macro particles with a simulation applying the 3D IMPACT code [7] with $10^{6}$ macro particles. Note that IMPACT with a 3D Poisson solver applied to the essentially $2 \mathrm{D}$ coasting beam problem should em- 
ploy a larger number of simulation particles than is used in MICROMAP with a 2D Poisson solver. The scope of the comparison with two sufficiently different numerical codes is to gain confidence in the particle-in-cell simulation for large turn number. Here we particularly worry about code-specific noise effects, which may be negligible on the time-scale of $10^{3}$ turns, but could have a significant effect over much longer times.

We first compare the output of simulation at the end of 1000 turns for the case of 200 A octupole current. At this level significant beam loss is expected, and we can thus test both, the emittance growth and dynamic aperture effect caused by the octupole. Note that we have chosen the slightly smaller space charge tune shift of $\Delta Q_{x}=0.075$ for this comparison. The result of Fig. 6 shows excellent agreement even in the dependence on $Q_{x}$. The detailed

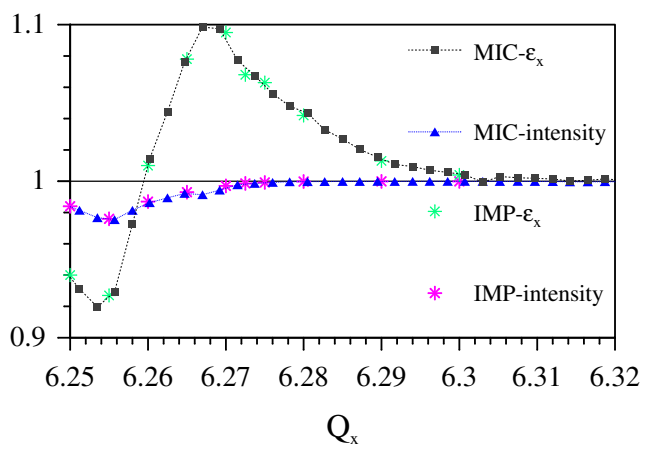

FIGURE 6. Comparison of MICROMAP and IMPACT simulation for 200 A octupole and $\Delta Q_{x}=0.075$ at 1000 turns.

evolution for $Q_{x}=6.27$ and 3200 turns (for MICROMAP 3400 turns) is shown in Fig. 7. It is noted that the rms emittances show a slow but steady growth after about 1000 turns, with at the same time a continuing small rate of loss. This steady growth cannot be attributed to just

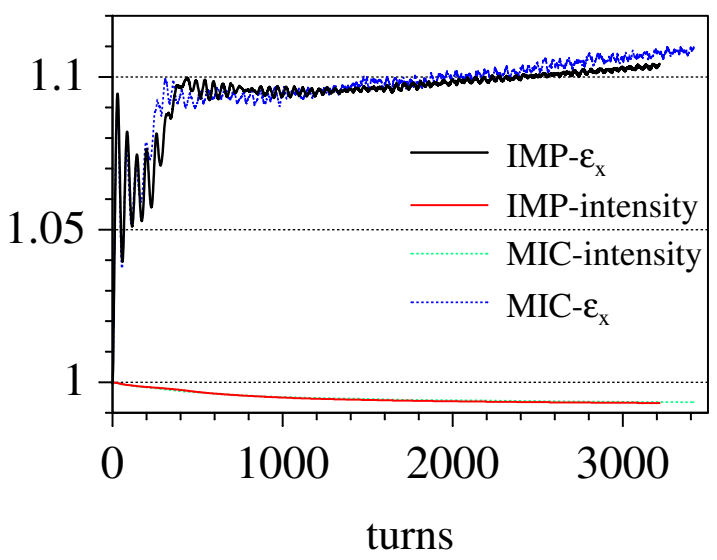

FIGURE 7. Evolution of MICROMAP and IMPACT simulations for $Q_{x}=6.27$.

simulation noise as it is completely absent if the octupole is turned off, in which case the relative emittance growth is less than $10^{-4}$. It may be associated with slow diffusion of particles into the dynamic aperture, hence these particles contribute to the rms emittance before they hit the simulation boundary. It must not be assumed that this process continues for ever on the same rate, since tails get depleted, and eventually the process slows down. This was supported by an extended run of this case up to $10^{4}$ turns with MICROMAP, where the growth was found to saturate at $13 \%$ beyond 8000 turns.

\section{CONCLUSION}

This study demonstrates that for Gaussian coasting beams, and under stationary external parameters, there is practically absence of coherent resonance effects, which justifies the use of non-self-consistent space charge calculation. The main reasoning behind this $2 \mathrm{D}$ finding is the effect of Landau damping due to the broad frequency spread of the Gaussian, which is even enhanced in bunched beams due to the additional spread from longitudinal current variation. This encourages the use of analytical space charge models for 3D studies, particularly in the realm of $10^{5}-10^{6}$ turns as use din Ref. [1], where fully self-consistent simulation appears impractical, except for special model tests on a reduced time scale. Obviously, such conclusions cannot be applied to a situation, where changing parameters (stacking injection, peak intensity increase during bunch rotation, shifting working point) enforce a significant dynamical change of the distribution function.

\section{ACKNOWLEDGMENTS}

This work was performed in part using resources of the NERSC scientific computing center of the US DOE.

\section{REFERENCES}

1. G. Franchetti et al. this conference.

2. A.V. Fedotov and I. Hofmann, Phys. Rev. ST Accel. Beams 5, 024202-1 (2002).

3. G. Franchetti, I. Hofmann, and G. Turchetti, AIP Conference Proceedings 448, 233 (1998).

4. A.V. Fedotov . R.L. Gluckstern and I. Hofmann, in Proceedings of the Particle Accelerator Conference, Portland, Oregon, 2003.

5. R. Baartman, AIP Conf. Proc. 448 (New York, 1998), p. 56.

6. I. Hofmann, G. Franchetti, O. Boine-Frankenheim, J. Qiang, R.D. Ryne, Phys.Rev.ST Accel.Beams 6:024202 (2003).

7. J. Qiang et al., J. Comp. Phys. 163, 434 (2000). 